\title{
Reduced Ventrolateral fMRI Response during Observation of Emotional Gestures Related to the Degree of Dopaminergic Impairment in Parkinson Disease
}

\author{
Martin Lotze ${ }^{1,2}$, Matthias Reimold ${ }^{2}$, Ulrike Heymans ${ }^{2}$, Arto Laihinen ${ }^{3}$, \\ Marianne Patt ${ }^{4}$, and Ulrike Halsband ${ }^{5}$
}

\begin{abstract}
Recent findings point to a perceptive impairment of emotional facial expressions in patients diagnosed with Parkinson disease (PD). In these patients, administration of dopamine can modulate emotional facial recognition. We used fMRI to investigate differences in the functional activation in response to emotional and nonemotional gestures between PD patients and age-matched healthy controls (HC). In addition, we used PET to evaluate the striatal dopamine transporter availability (DAT) with $\left[{ }^{11} \mathrm{C}\right] \mathrm{D}$-threo-methylphenidate in the patient group. Patients showed an average decrease to 26\% in DAT when compared to age-corrected healthy references.
\end{abstract}

\section{INTRODUCTION}

Parkinson disease (PD), one of the most prevalent neurological disease in industrial countries (Hirtz et al., 2007), is associated predominantly with motor impairment, with reduced facial and gesture expression as key symptoms (Smith, Smith, \& Ellgring, 1996). The motor impairment often is accompanied by neuropsychological deficits (Dubois \& Pillon, 1997), including abnormalities in emotional processing, such as impaired perception of vocal material [prosody (Pell, 1996; Blonder, Gur, \& Gur, 1989); vocal emotions (Breitenstein, Van Lancker, Daum, \& Waters, 2001)], as well as visual recognition of emotional (Suzuki, Hoshino, Shigemasu, \& Kawamura, 2006; Kan, Kawamura, Hasegawa, Mochizuki, \& Nakamura, 2002; Breitenstein, Daum, \& Ackermann, 1998) and nonemotional faces (Dewick, Hanley, Davies, Playfer, \& Turnbull, 1991). Selective deficits in recognition of facial expressions of disgust are observed in symptomatic PD patients as well as in patients with presymptomatic Huntington's disease, an illness associated with neuronal loss in the striatum (Suzuki et al., 2006; Hennenlotter et al., 2004).

${ }^{1}$ University of Greifswald, Germany, ${ }^{2}$ University of Tübingen, Germany, ${ }^{3}$ Neuro-Rehamed GmbH, Stuttgart, Germany, ${ }^{4}$ University of Leipzig, Germany, ${ }^{5}$ University of Freiburg, Germany
Reduction in the DAT of the left putamen correlated not only with motor impairment but also with errors in emotional gesture recognition. In comparison to HC, PD patients showed a specific decrease in activation related to emotional gesture observation in the left ventrolateral prefrontal cortex (VLPFC) and the right superior temporal sulcus. Moreover, the less DAT present in the left putamen, the lower the activation in the left VLPFC. We conclude that a loss of dopaminergic neurotransmission in the putamen results in a reduction of ventrolateral prefrontal access involved in the recognition of emotional gestures.
The most important pathology in PD is the loss of dopaminergic neurons in the substantia nigra, resulting in significantly reduced dopamine transporter availability (DAT) predominantly in the striatum. To assess dopaminergic loss with positron emission tomography (PET) or single photon emission computed tomography (SPECT), various radioligands have been developed to characterize presynaptic function. Dopamine synthesis ([18F]fluorodopa), dopamine transporter availability (various ligands) and vesicular monoamine transporter availability ([11C]dihydrotetrabenezine) are all reduced in Parkinson disease and were recently compared in the same individuals (Sossi et al., 2007). While, on a synaptic level, opposed effects may occur (e.g., downregulation of DAT resulting in increased synaptic dopamine), all of these tracers show the gradual loss of dopaminergic neurons over the course of Parkinson disease. For clinical diagnosis of Parkinson, the most frequently applied tracers are ligands of the dopamine transporter. Among these, [11C]Dthreo-methylphenidate (Volkow et al., 1995) is a PET tracer that allows to accurately and selectively evaluate DAT in regions with high density of dopamine transporters (striatum). Recently, a rather close correlation between reduced striatal binding of this tracer and motor impairment in patients with Parkinson disease was described (Breit, Reimold, Reischl, Klockgether, \& Wullner, 2006). 
The striatum, including the caudate and the putamen, has an important function for the execution of involuntarily motor response, such as spontaneous facial expression (Yin \& Knowlton, 2006). With respect to the perception of facial expression, research using functional imaging revealed an important involvement of the putamen in monkey (Logothetis, Guggenberger, Peled, \& Pauls, 1999) and man (Phillips et al., 1998). Lesions of the basal ganglia result in decreased recognition of emotional facial expression of anger, disgust, and fear (Cheung, Lee, Yip, King, \& Li, 2006), and there is anecdotal evidence that lesions of the left putamen and insula result in impairment of recognition of the facial expression of disgust (Calder, Keane, Manes, Antoun, \& Young, 2000).

The deteriorating effect on emotional facial recognition by a lesion of the putamen might be caused by a pathway connecting facial recognition areas of the inferior and medial-temporal lobe with emotional appraisal areas located in the ventrolateral prefrontal cortex (VLPFC; BA 47) via the putamen. This pathway has been demonstrated by using tracing techniques in the macaque brain (Cavada, Company, Tejedor, Cruz-Rizzolo, \& Reinoso-Suarez, 2000; Middleton \& Strick, 1996). For the inferior temporal lobe, the fusiform gyrus is crucial for the processing of facial recognition (Shmuelof \& Zohary, 2005; Haxby, Hoffman, \& Gobbini, 2000). In the medial-temporal lobe, the region around the superior temporal sulcus (STS) is crucially involved in social recognition which, for the visual domain, can be composed of inputs from different body parts such as the face including eye and mouth, hands, and postures of the body stem of another person or animal (Allison, Puce, \& McCarthy, 2000). The STS is, therefore, especially important for the processing of social gestures such as expressive gestures, and its activity is modulated by the emotional content of these gestures (Lotze et al., 2006). The VLPFC is involved both in the emotional appraisal of visual (facial recognition; Levesque et al., 2003; Sprengelmeyer, Rausch, Eysel, \& Przuntek, 1998) and auditory material (recognition of prosody; Wildgruber et al., 2005). A destruction of this connection might result in impaired emotional recognition as the information of facial and social recognition and those of emotional recognition of facial expression cannot be integrated anymore.

A recent publication explores the interconnection between lesions of the limbic system (amygdala) and the effect of L-DOPA on the emotional recognition of faces (Sato, Takahashi, \& Kawamura, 2008). The authors demonstrated that impaired facial recognition scores for the expression of fear and disgust in one patient with amygdala lesions was changed to normal recognition scores after oral L-DOPA medication. This anecdotal data suggest that dopamine availability and lesions of the emotional recognition network are associated.

In patients suffering from PD, the striatum shows a reduction of DAT. It might be conceivable that dopami- nergic activity in the putamen does not only modulate the emotional recognition of faces in an unspecific manner, as it has been demonstrated by an application of a dopamine D2-antagonist in healthy subjects, which caused an impaired recognition of angry faces (Lawrence, Calder, McGowan, \& Grasby, 2002). More specifically, dopaminergic activity in the putamen might modulate the pathway between areas processing analytic facial recognition in the fusiform gyrus, the STS, and the VLPFC associated with the appraisal of multiple emotional inputs. To investigate this possible effect in PD patients, we used $\left[{ }^{11} \mathrm{C}\right] \mathrm{D}$ threo-methylphenidate to selectively evaluate striatal DAT in these patients. Additionally, we measured the blood oxygenation level-dependent (BOLD) effect in these patients in response to emotional and nonemotional body and face referred meaningful gestures. We hypothesized that the left VLPFC, which has been shown to be specifically involved in the emotional recognition of gestures (Lotze et al., 2006), should be modulated in their fMRI activation by the availability of dopamine within the putamen. In order to detect an overall effect of impaired cerebral activation during observation of emotional and nonemotional expressive gestures, we compared the BOLD magnitude in preselected regions known to be involved in the processing of these gestures between PD patients and age-matched controls.

\section{METHODS}

\section{Subjects and Procedure}

PD patients were recruited via letters to regional selfhelp groups or by referral from associated neurologists. Complete disease history, inventory of medications and symptoms, as well as clinical neurologic evaluations, were conducted for each of these patients. Ten patients with diagnosed idiopathic PD and 10 age-matched healthy controls (HC) gave written informed consent. After an additional neurologic examination, one patient presented an unusual rapid development of PD symptoms and he was diagnosed with multisystem atrophy. This patient and another age-matched $\mathrm{HC}$ were excluded from further analysis. The nine idiopathic PD patients remaining ( 1 woman; mean age $=65.7 \pm$ 3.8 years) showed a large range in disease duration (521 years; mean $=12.8 \pm 4.1$ years $)$. PD severity $[$ Hoehn and Yahr score (HY; Hoehn \& Yahr, 1967); median = 2], Unified Parkinson's Disease Rating Scale (UPDRS) (Fahn \& Elton, 1987; mean $=38.38 \pm 14.30)$, cognitive impairment [Mini-Mental State Exam (MMS; Folstein, Folstein, \& McHugh, 1975); mean: $29.67 \pm 0.67]$, and mood disturbances [Beck Depression Inventory (BDI II; Beck, Ward, Mendelson, Mock, \& Erbaugh, 1961); mean = $8.67 \pm 2.04]$ were assessed during the off-state of medication before the fMRI measurements. fMRI and PET measurements were within a range of 10 days. Medication in all patients included levodopa; seven took carbidopa, five 
took amantadine, and three took cabergoline, in addition. Nine healthy age-matched controls (HC, 3 women; mean age $=62.7 \pm 4.8$ years) were recruited by announcements posted at the University and a local adult education center. HC subjects lacked any history of neurological or psychiatric disease. Demographic and clinical data of patients are provided in Table 1. Patients and controls were investigated according to their handedness and all were strongly right handed (Oldfield, 1971). The study was approved by the ethics committee of the Medical Faculty of the University of Tübingen. Written informed consent was obtained according to the guidelines of the Declaration of Helsinki.

\section{PET Scanning Parameters}

Patients were asked to cease taking medication the evening before PET measurements. Thus, all patients were drug-free for more than 12 hours before PET examination. We used $\left[{ }^{11} \mathrm{C}\right] \mathrm{D}$-threo-methylphenidate $(\mathrm{dMP})$ as a biomarker for the progression of PD. This tracer allows for accurate noninvasive quantification of the binding potential in regions with a high density of dopamine transporters (Logan et al., 1996) (binding potential, $\mathrm{BP}_{2}$ ) and is insensitive to synaptic dopamine levels (Gatley et al., 1995). Recently, we showed that the striatal binding potential of dMP correlates closely with motor symptoms and disease duration (Breit et al., 2006), and thus, can be considered a good biomarker of progression of PD. DMP synthesis, PET scanning, and data analysis are described in detail in an earlier work (Breit et al., 2006). For PET scanning, the patient's head was fixed in an elastic mold with adhesive tape. Three fiducial markers were attached to the skull to support for correction of head movements. After automated intravenous bolus injection (12 sec) of $700 \mathrm{MBq}$ dMP, dynamic data were acquired 0 to 60 min postinjection (pi) in 2-D mode with a full-ring PET scanner (GE Advance, General Electric Medical Systems, Milwaukee, USA), followed by a transmission scan with 500,000 kcts for attenuation correction. Attenuation corrected images were reconstructed with filtered back-projection $(128 \times 128$ pixel corresponding to $30 \times 30 \mathrm{~cm}$, Hanning filter with $4.6 \mathrm{~mm}$ cutoff).

\section{Evaluation of PET data}

The statistical parametric mapping software package (SPM99) was used for realignment and spatial normalization by comparing summation images 0 to 5 min pi with the standard SPM perfusion template. Time-activity curves of dMP were derived from MNI ROIs in the putamen $(2 \times$ $0.67 \mathrm{ml}$ ). For each patient, ROIs were compared visually with normalized images 0 to $5 \mathrm{~min}$ pi and optionally shifted up to one pixel $(2 \mathrm{~mm})$ in each dimension. For quantification of DAT, we calculated the binding potential $\mathrm{k} 3 / \mathrm{k} 4$ (denoted BPND in the recent consensus nomenclature,

Table 1. Behavioral Scores and PET measures of Parkinson's Disease Patients

\begin{tabular}{|c|c|c|c|c|c|c|c|c|c|}
\hline \multirow[b]{2}{*}{$A g e^{a}$} & \multirow{2}{*}{$\begin{array}{c}\text { Illness } \\
\text { Duration }^{a}\end{array}$} & \multirow{2}{*}{$\begin{array}{l}\text { Recognition } \\
\text { Mistakes }^{b}\end{array}$} & \multirow[b]{2}{*}{$M M S^{c}$} & \multirow[b]{2}{*}{ Total UPDRS ${ }^{d}$} & \multirow[b]{2}{*}{ Motor UPDRS } & \multirow{2}{*}{$\begin{array}{c}\text { Beck's } \\
\text { Depression }^{f}\end{array}$} & \multirow[b]{2}{*}{$H Y^{g}$} & \multicolumn{2}{|c|}{$D A T^{b}$} \\
\hline & & & & & & & & put le & put ri \\
\hline 63 & 12 & 7 & 30 & 45 & 20 & 6 & 3 & 22.13 & 18.50 \\
\hline 68 & 13 & 10 & 28 & 57 & 27 & 6 & 4 & 16.88 & 12.81 \\
\hline 61 & 12 & 7 & 29 & 40 & 14 & 4 & 2 & 22.20 & 21.43 \\
\hline 72 & 14 & 5 & 30 & 46 & 16 & 5 & 2 & 26.30 & 31.99 \\
\hline 67 & 16 & 3 & 30 & 18 & 8 & 4 & 1 & 27.43 & 20.57 \\
\hline 62 & 10 & 2 & 30 & 35 & 12 & 6 & 1 & 23.74 & 21.10 \\
\hline 61 & 12 & 4 & 30 & 32 & 13 & 17 & 3 & 20.05 & 14.48 \\
\hline 68 & 21 & 5 & 30 & 57 & 19 & 21 & 4 & 18.99 & 13.71 \\
\hline 69 & 5 & 6 & 30 & 19 & 8 & 9 & 1 & 23.74 & 13.18 \\
\hline
\end{tabular}

${ }^{\mathrm{a}}$ Years since first symptoms which resulted in the PD diagnosis.

${ }^{\mathrm{b}}$ Mistakes in gesture recognition in absolute values.

${ }^{\mathrm{c}}$ Mini Mental State; optimal score: 30.

dUnified Parkinson's Disease Rating Scale; all scores.

'Unified Parkinson's Disease Rating Scale; only motor scores.

f Beck's Depression Scale.

${ }^{\mathrm{g}}$ Hoehn and Yahr score.

${ }^{\mathrm{h}}$ Dopamine transporter availability as measured with PET; put = putamen; le = left; ri = right. 
see Innis et al., 2007), a measure proportional to the density of dopamine transporters), using Logan's graphical analysis and the occipital cortex as reference region (washout $k_{2 \mathrm{R}}=0.05 \mathrm{~min}^{-1}$, regression interval $=18-60 \mathrm{~min} \mathrm{pi}$; Logan et al., 1996). Age-corrected values for DAT were expressed in $\%$, assuming a loss per decade of $6.6 \%$, fitted to a previous control group (Breit et al., 2006).

\section{fMRI Measurements}

Patients were asked to cease taking medication the evening before fMRI measurements. Thus, all patients were drug-free for more than 12 hours before fMRI. All subjects were screened verbally for ametropia, and deficits in vision of more than +1 diopter were corrected with MR-compatible glasses. Volunteers were placed in a supine position on the padded scanner couch and video clips were displayed via a mirror fixed on the head coil. The videos comprised a set of meaningful gestures: Clips with emotional and nonemotional gestures both showed hand movements together with the actor's face and upper half of the body (see Figure 1). The duration of each video was $3 \mathrm{sec}$ with a frame rate of 30 pictures per second. A gray field of the same size as the videos with the average luminance of all clips was shown between presentations of the video clips (baseline). More detailed information for imaging, presented material, and data evaluation are given in a previous study on a larger group of healthy subjects (Lotze et al., 2006). Subjects were asked to keep their eyes open and look at the mirror to view the video. They were instructed to observe the gestures without imitating them and without moving their hands or face. Subjects showed no visible movements of their hands during gesture presentation. Whole head scans were performed with a 1.5-Tesla whole body scanner (Siemens Vision) using echo-planar imaging (EPI; $\mathrm{TE}=40 \mathrm{msec}$; $\mathrm{TR}=3 \mathrm{sec} ; 28$ axial slices of $4 \mathrm{~mm}$ slice thickness and $1 \mathrm{~mm}$ gap; matrix $=64 \times 64$ ). In an event- related design, the 30 different video clips were presented in a pseudorandomized order with each clip appearing twice. Overall, total measurements comprised 360 EPI datasets per subject. The intersession interval (ISI) was $18 \mathrm{sec}$ in order to permit recognition of the main effect of each gesture and identify differences between gesture types. Measurements, including the discarded images, were conducted in five blocks each lasting for approximately $4 \mathrm{~min}$. The video material was rated verbally after fMRI measurements. The subject was watching the same video clips as he or she observed in the MR scanner in a randomized order and verbally described the meaning of the presented clip (e.g., "threatening with a fist"), and a scientist (U. H.) documented the verbal comments. Additionally, emotional gestures were rated for their valence using a visual analogue scale (VAS) ranging from -5 (emotional negative) to 0 (neutral) to +5 (emotional positive). These data were evaluated for correctness (false responses in percent of all) and emotional valence (infinitely variable between 0 and 10). Type of errors were classified as mistaking emotional for nonemotional gestures and as mistaking one emotion for another.

\section{Evaluation of fMRI data}

The first three EPI datasets of each session were discarded prior to analysis to allow for T1-saturation effects. Data were analyzed with the Statistical Parametric Mapping Program (SPM2; Wellcome Department of Imaging Neuroscience) running on Matlab version 7.1 (MathWorks, Natick, MA). Each individual scan was realigned to the first scan of a given condition to correct for movement artifacts. The realigned data were spatially normalized to the MNI template and resliced at $3 \times 3 \times 3 \mathrm{~mm}$. The resulting images were smoothed with a $9 \times 9 \times 9 \mathrm{~mm}$ (full width at half maximum) Gaussian filter. To correct for low-frequency components, a high-pass filter with a cutoff of 1/90 Hz was used. Statistical analyses were
Figure 1. Description and illustration of the video material presented.
A Nonemotional gestures:

Gestures of the right hand in relation to the face and the upper trunk:

e.g., brushing one's teeth, combing hair, shaving, blowing nose, cleaning glasses, creaming face.

\section{B Emotional gestures:}

Gestures of the right hand presented together with the face and the upper trunk:

e.g., whistling friendly, joking with somebody, threatening with the fist; threatening with the index finger, giving somebody the finger, showing you are silly.

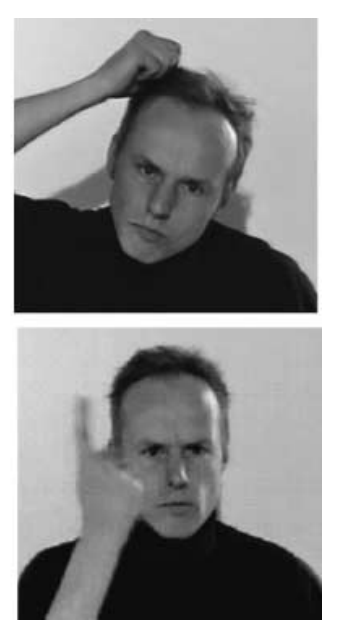


performed using the general linear model as implemented in SPM2. For each participant, a design matrix was created using a canonical hemodynamic response function for each of the two stimulus conditions. The movement parameters estimated during the realignment procedure were introduced as covariates into the model. Individual statistical maps (fixed effect) were calculated for each type of gesture (main effect for nonemotional and emotional gestures) and for interactions between gesture type. Corresponding contrast images of each subject were then entered into a second-level random effects analysis, which accounts for the variance between subjects. Activation maps (threshold $p<.05$; false discovery rate [FDR]; Genovese, Lazar, \& Nichols, 2002) of a pilot study in 20 HC subjects using the same stimulus material and the same fMRI scanner (Lotze et al., 2006) were used to generate masks related to the different gesture types. These masks comprised 5574 voxels for the emotional gesture condition, 6887 voxels for the nonemotional gestures, and 99 voxels for the comparison emotional minus nonemotional gestures. We used a multiple comparison correction within these masks $(p<$ .05 , FDR) for evaluations of significant main effects, interactions, and differences between subject groups. Highest activated voxels per condition were assigned to anatomical regions and given in MNI coordinates. All anatomical regions were detected with the "Automated Anatomical Labeling" software (AAL) (Tzourio-Mazoyer et al., 2002); regions already mapped cytoarchitecturally (BA 4, BA 6, BA 7, BA 44, amygdala) were assigned to Brodmann's areas (50\% probability) using ANATOMY (Eickhoff et al., 2005). Both software packages are integrated as a toolbox in SPM2.

\section{Correlations of DAT with Other Data Parameters}

The correlation between the number of errors in emotional gesture recognition and fMRI activation during this condition was performed with SPM2 regression analysis within areas associated with the processing of emotional gestures in the prestudy $(p<.05$, FDR). For the correlation analysis between DAT and fMRI response, we used a more restricted analysis: From the prestudy we knew that the VLPFC of the left hemisphere is involved more specifically in the processing of emotional gestures. Therefore, we performed a correlation analysis between fMRI BOLD values within left VLPFC activation and putamen DAT. For this correlation, we used the activation map specific for observation of emotional gestures and averaged the BOLD magnitude within a sphere of $3 \mathrm{~mm}$ around the highest activated voxel (parameter estimates; see Figure 3). Correlations of DAT with clinical scores (HY; UPDRS) and number of errors in gesture recognition were calculated using one-sided tests, as we expected a negative correlation of performance with DAT. All correlations were performed with the Statistical Package for the Social Sciences, version 10.05.

\section{RESULTS}

\section{Performance Data}

PD patients made more gesture recognition errors $(M=$ $13.89 \%, S D=6.01)$ than $\mathrm{HCs}[M=4.72 \%$; $S D=2.32$; $t(16)=4.14, p<.001]$. Emotional gestures specifically were recognized at a reduced rate in patients $[t(16)=$ 2.89, $p<.05$; patients: $M=24.66 \%, S D=17.32$; HCs: $M=7.11 \%, S D=5.63]$, whereas the rate of recognition of nonemotional gestures was not significantly decreased $[t(16)=1.92, n s$; patients: $M=20.11 \%, S D=$ 19.49; HCs: $M=7.28 \%, S D=5.63]$. A more detailed comparison of the type of misattribution in the patients' group demonstrated that those for mistaking emotional for nonemotional gestures were 4.83 times more often than those for mistaking one emotion for another $[t(8)=$ $2.60, p<.05$; see Figure 2]. However, valence ratings did not differ between subject groups $[t(16)=0.35, n s]$ nor were any significant subject group differences in valence ratings for positive, negative, or neutral stimuli identified. The Mini Mental State (MMS) scores did not differ between patient and control groups $[t(16)=1.41, n s]$.

\section{Dopamine Transporter Availability}

DAT of PD patients was reduced to $22.38 \pm 3.39 \%$ in the left putamen of previously reported control levels (Breit et al., 2006). The HY score of PD severity was inversely correlated with DAT in the left putamen $(r=-.87, p<$ .001). In addition, motor impairment in UPDRS correlated negatively with left putamen DAT $(r=-.73, p<$ $.05)$. DAT in the right putamen was lower than those within the left putamen [paired $t$ test: $t(8)=2.50, p=$ .037]. However, motor symptoms-as assessed with the UPDRS - were not significantly lateralized. Right-sided motor symptoms correlated with left putamen DAT $(r=$ $.80, p<.005)$. With respect to gesture recognition, left

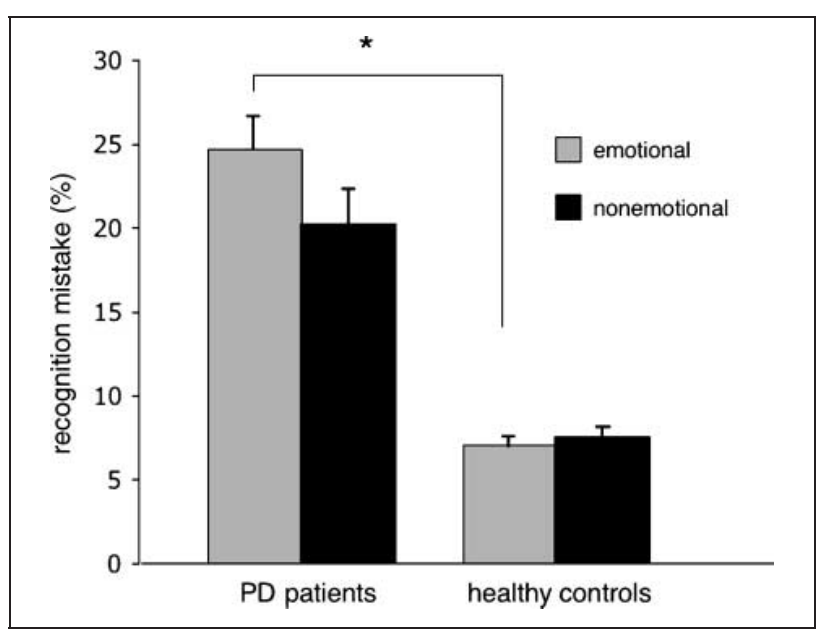

Figure 2. Percent recognition mistakes in PD patients and healthy controls for emotional (gray) and nonemotional (black) gestures. Bars indicate standard error of recognition mistakes. 
putamen DAT correlated negatively with the number of errors (one sided; $r=.60, p<.05$ ).

\section{fMRI}

fMRI responses to emotional gestures in age-matched HCs showed a comparable activation pattern to that described for a larger group of younger subjects (see Lotze et al., 2006). Cortical responses specific to the emotional gestures were obtained by subtracting activation maps observed during nonemotional gestures from those obtained during emotional gestures. Activation sites specific for emotional gestures were located in the left BA 47, the superior frontal gyrus (BA 6 and 8), the right cerebellar hemisphere, the right STS, and the right temporal pole (Table 2A). The same contrast for patients revealed activation in the left superior temporal gyrus and the left VLPFC (Table 2B). PD patients showed reduced fMRI activation in multiple areas during observation of all presented gestures. For the nonemotional gestures, these comprised the bilateral occipito-temporal junction including the visual motion area (V5), the fusiform gyrus, regions around the bilateral intraparietal sulcus, the right dorsal premotor cortex, the supplementary motor area (SMA), the cerebellar hemispheres, the right STS, the temporal poles, and the left putamen (see Figure 3A and Supplementary Table 1A). When brain activation during observation of emotional gestures was compared between groups, PD patients showed lower activation in the following additional areas: left BA 47, left STS, bilateral sensorimotor cortex, bilateral putamen, medial cingulate gyrus, midbrain, and bilateral amygdala (see Figure 3B and Supplementary Table 1B). Activation in the left BA 47 and the right STS, in response to the recognition of emotional gestures, was reduced specifically in PD patients (Table 2C); however, PD patients showed no significant increase of activation for all comparisons with HCs.

\section{Correlation of fMRI and DAT data}

The number of errors in recognition of emotional gestures by PD patients correlated with increased activation in the bilateral insula (right: $t=7.31, p_{\mathrm{FDR}}=.028$, cOordinates $4215-12$; left: $t=5.40, p_{\mathrm{FDR}}=.046$, coordinates $-4512-9)$. In contrast, the number of errors in PD-patient recognition of nonemotional gestures correlated with fMRI activation in the left STS (coordinates $-54-423, t=6.67, p<.001$ ) and in the left (coordinates $-30-7524, t=5.80, p<.001)$ and right MT (coordinates $30-7527, t=4.50, p<.001$ ). Left BA 47 activation for the contrast emotional minus nonemotional gestures was positively correlated with left putamen DAT $(t=4.52$, coordinates $-4230-12, r=.70$, $p=.016 ;$ Figure 4).

\section{DISCUSSION}

In this study, we investigated the differences in cerebral representation specific to emotionally relevant gestures in patients affected with PD in comparison to agematched control subjects. In addition, we wanted to identify correlates of BOLD magnitude associated with impaired recognition of emotional and nonemotional gestures and with the availability of dopamine in the

Table 2. Activation Sites Specific for Emotional Gestures

\begin{tabular}{|c|c|c|c|c|}
\hline Area & $t$ & \multicolumn{3}{|c|}{ MNI Coordinates } \\
\hline \multicolumn{5}{|c|}{ (A) Healthy Control Subjects: Emotional Gestures Minus Nonemotional Gestures } \\
\hline Ventrolateral prefrontal left (VLPFC, BA 47) & 12.12 & -54 & 24 & -4 \\
\hline Superior frontal gyrus left (BA 8) & 7.89 & -3 & 33 & 57 \\
\hline SMA (BA 6) & 5.67 & 0 & 12 & 63 \\
\hline Cerebellar hemisphere right & 5.23 & 24 & -84 & -33 \\
\hline Superior temporal sulcus right (STS; BA 22) & 4.47 & 51 & -21 & -12 \\
\hline Temporal pole right (BA 38) & 3.48 & 51 & 12 & -33 \\
\hline \multicolumn{5}{|c|}{ (B) PD patients: Emotional Gestures Minus Nonemotional Gestures } \\
\hline Superior temporal gyrus left (BA 22) & 6.77 & -60 & -51 & 18 \\
\hline Ventrolateral prefrontal left (VLPFC, BA 47) & 3.81 & -45 & 27 & -12 \\
\hline \multicolumn{5}{|c|}{ (C) Healthy Control Subjects Minus PD Patients: Emotional Gestures Minus Nonemotional Gestures } \\
\hline Ventrolateral prefrontal left (BA 47) & 3.87 & -54 & 24 & -9 \\
\hline Superior temporal sulcus right (STS; BA 22) & 3.39 & 51 & -24 & -9 \\
\hline
\end{tabular}




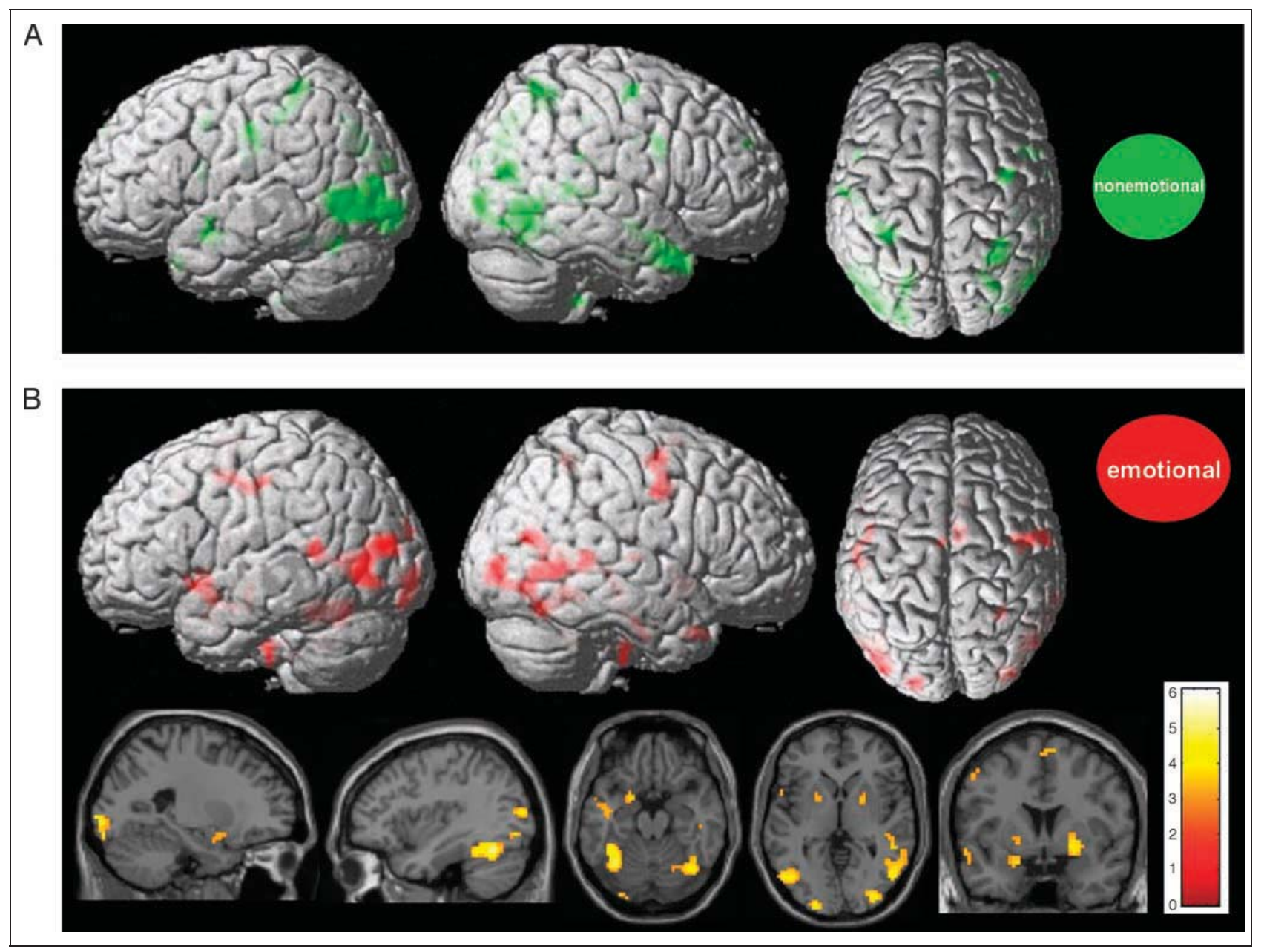

Figure 3. Healthy control subjects minus Parkinson's disease patients; Activation maps are projected on the MNI reference brain as provided in SPM2. Activation maps are thresholded with $p<.05$ and false discovery rate (FDR) within activated voxels of the same contrast of a healthy pilot group using the same stimulus material and the same fMRI scanner (Lotze et al., 2006). (A) During observation of nonemotional gestures (green), healthy subjects showed increased activation in bilateral visual, cerebellar, premotor areas, the posterior parietal lobe, the left putamen (not visible here), the bilateral opercular part of the inferior frontal gyrus (BA 44), the bilateral anterior parietal lobes, the temporal poles, the superior temporal sulci (STS), and the brainstem (not visible). Detailed activation sites are given in Supplementary Table 1A. (B) During observation of emotional gestures (red), healthy subjects showed increased activation in the primary sensorimotor cortex, the pre-SMA, the left ventrolateral prefrontal lobe (BA 47), the bilateral amygdalae and putamen (shown in the slices below), the medial cingulate cortex, and the midbrain (not visible). Detailed activation sites are given in Supplementary Table 1B.

putamen. We hypothesized that these changes would be localized in regions also involved in the processing of emotional and nonemotional gestures of healthy subjects (Lotze et al., 2006). Therefore, we used fMRI to measure a group of idiopathic PD patients with different levels of severity of motor disability who were medication-free for more than 12 hours, and a group of age-matched controls who observed emotional and nonemotional gestures. In the patient group, we also measured DAT in the putamen in order to quantify the dopaminergic deficit. DAT in the putamen was reduced to $26 \%$, on average, in comparison to healthy subjects of the same age (Breit et al., 2006).

We detected substantial deficits of cerebral activation in patients diagnosed with idiopathic PD in compari- son to healthy age-matched controls during observation of nonemotional and emotional gestures. Specifically, for emotional gestures, the left VLPFC and the right STS showed lower activation in PD patients.

VLPFC (BA 47) activity has been correlated with a number of different emotional sensations, including sad feelings associated with watching sad movies (Levesque et al., 2003). BA 47 activation has been described to be critically involved in the emotional processing of visual (facial recognition; Levesque et al., 2003; Sprengelmeyer et al., 1998) and auditory material (recognition of prosody; Wildgruber et al., 2004). Lesions within the VLPFC decrease recognition of both facial and vocal emotional expression (Hornak, Rolls, \& Wade, 1996). In a larger group of younger healthy subjects, we demonstrated 
Figure 4. Correlation of fMRI activation magnitude during observation of emotional gestures minus nonemotional gestures in PD patients with PET dopamine transporter availability (DAT; age-corrected values expressed in $\%$; $x$-axis) in the left putamen. The activation map specific for emotional gestures projected on the MNI single-subject segmented brain was masked with the region of interest from the prestudy (Lotze et al., 2006). Beta values ( $y$-axis) of the BOLD magnitude in the left BA 47 (average of $3 \mathrm{~mm}$ sphere around the highest activated voxel; coordinates: -4230 -12 ) are plotted against left putamen DAT ( $x$-axis), showing a positive correlation of $r=.70$.

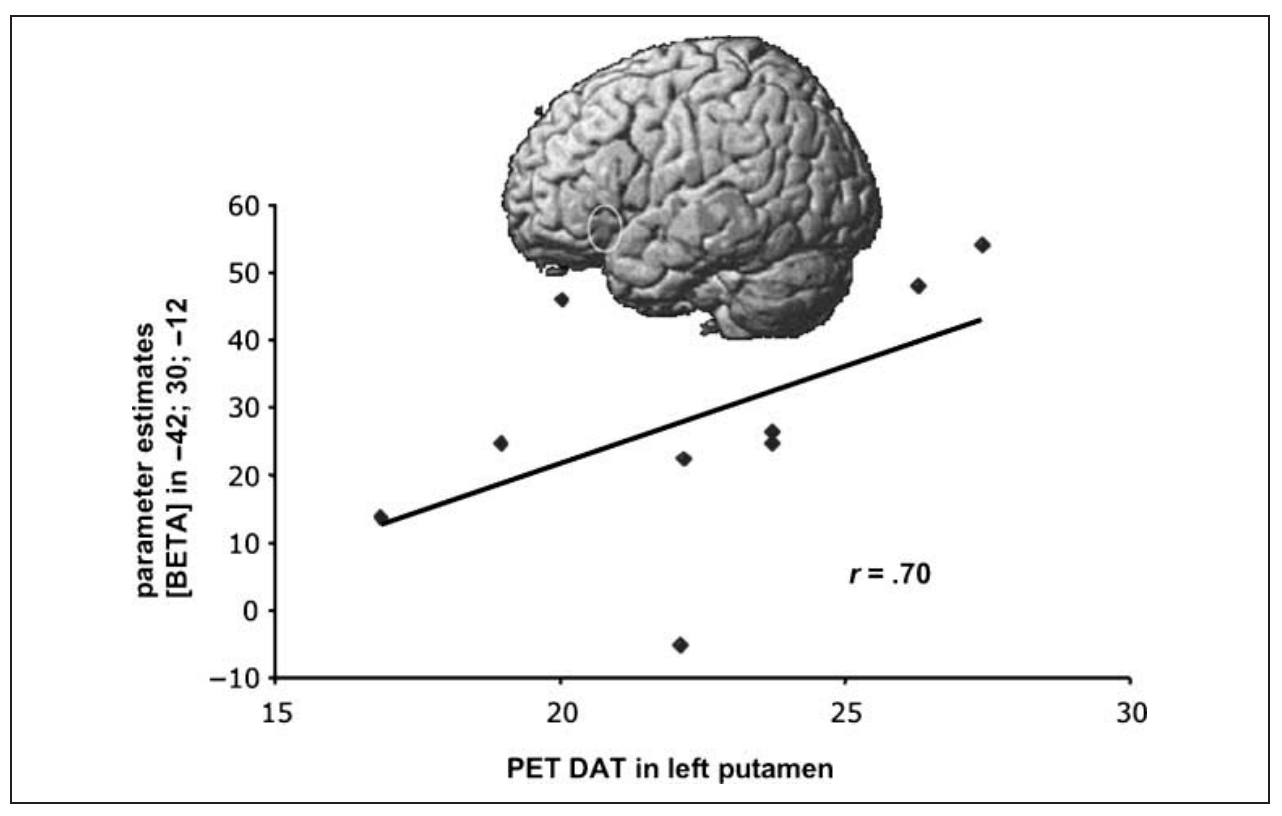

previously that the VLPFC is crucial for the processing of emotional gestures (Lotze et al., 2006). In the present study, we tested the criteria specific to the observation of emotional gestures by comparing activation maps assessed during observation of emotional gestures minus those assessed during nonemotional gestures. This contrast revealed activation in the left BA 47 only in HCs but not in PD patients. Consistently, left BA 47 activation differed between subject groups.

In line with other studies that reported impaired emotional recognition in PD patients (Lawrence, Goerendt, \& Brooks, 2007; Suzuki et al., 2006; Sprengelmeyer et al., 2003), we found a significant decrease of recognition of emotional but not nonemotional gestures in the PD patients. With respect to the specific recognition deficits of emotional gestures, we hypothesized that a decrease in left BA 47 activation should correlate with decreased left putamen DAT, which indeed was the case; access to BA 47 seems to be modulated by dopaminergic activity in the left putamen.

Facial and social recognition areas in the temporal lobe are connected to the ventral striatum (Middleton \& Strick, 1996), and the ventral striatum projects into the VLPFC (Alexander \& Crutcher, 1990). Loss of ventral striatal dopaminergic activity might therefore specifically modulate the interconnection between analytic temporal facial recognition areas (fusiform gyrus and STS) and an area of prefrontal appraisal such as the VLPFC. Predominantly, three findings in this study support this assumption: Firstly, only activation in the VLPFC was differentially significant when emotional minus nonemotional gestures were calculated in healthy subjects. Secondly, VLPFC activation within the left hemisphere was significantly increased if the interaction between emo- tional and nonemotional gesture observation was compared between HCs and PD patients. Thirdly, DAT in the left putamen and left VLPFC BOLD magnitude correlation was significantly positive.

However, more unspecific dopamine effects on emotional recognition cannot be excluded. Interestingly, patients made more mistakes misinterpreting emotional to nonemotional gestures than in misinterpreting one emotion for another. Because the expression of the face is an important part of the emotional gesture presented and there was no significant increase in the recognition of nonemotional gestures, we conclude that the main effect of misinterpretation of emotional gestures is probably in the missing recognition of emotional facial expression. This is presumably processed in the VLPFC.

Our findings with respect to the dependence of gesture recognition on putamen DAT, recognition deficits, and access to the VLPFC need replication with larger sample populations. Furthermore, it would be extremely interesting to expand these correlative findings with a dopamine stimulation study: If substitution of dopamine modulates BA 47 activation during observation of emotional gestures, a causal connection between dopamine and activation in BA 47 could be assumed.

It must be considered that PD also affects structures other than the basal ganglia and the amygdala, such as the insula, the entorhinal region, the hippocampus, the thalamus, and the anterior cingulate (Braak et al., 1996). All of these areas might contribute to altered recognition of emotional gestures. A previous study measuring the fMRI response to angry and fearful faces before and after dopamine substitution revealed a significant increase in amygdala activation, which was the only ROI in that study (Tessitore et al., 2002). These authors used a 12-hr hypo- 
dopaminergic state as baseline, which was comparable with our investigations in dopamine-deprived patients.

In our study, the bilateral anterior insula showed increased activation with an increasing amount of errors in emotional gesture recognition in PD patients. Together with the bilateral amygdala, the anterior cingulate cortex, and the medial and lateral ventral prefrontal cortex, this area is involved in emotional processing of pictures (Phan, Wager, Taylor, \& Liberzon, 2002). The insula is associated with the subjective feeling of emotional states. The anterior insula seems to reflect the self-conscious aspect of the rated valence of a given emotion (Anders, Lotze, Erb, Grodd, \& Birbaumer, 2004). Consistent with the importance of the anterior insula for the valence dimension, patients involved in our study showed no decrease in valence ratings of emotional stimuli and no decrease of insula activity in comparison to HCs. Reports about reduced dorsoanterior insula activity in Huntington's disease patients during perception of faces with the expression of disgust (Hennenlotter et al., 2004) underline the fact that the insula is differentially involved in this basic emotion, a finding also demonstrated in patients with selective lesions of the insula and the striatum (for a review, see Calder, Lawrence, \& Young, 2001). It has to be kept in mind that Huntington's disease involves a loss of different neurotransmitters than that observed in PD (Bernheimer, Birkmayer, Hornykiewicz, Jellinger, \& Seitelberger, 1973), which results in different deficits, and might therefore also involve different compensation strategies. For PD patients, it seems plausible that with increasing problems in recognition of emotional gestures, the insula is increasingly involved, possibly compensating for a loss of dopamine in the putamen.

In contrast, only the bilateral visual motion area (MT) and the left STS correlated with problems in recognizing nonemotional gestures. These data underline that areas involved in the analysis of movements and gestures are increasingly active when having difficulties with the appropriate assignment of the nonemotional gestures and that correlates of recognition mistakes in nonemotional material are restricted to these analytic areas and do not involve prefrontal regions or regions involved in emotional processing.

In the patient group, BOLD magnitude, which is specific to emotional gestures, was not only decreased in the left VLPFC, but also in the right STS. The STS is a polysensory area, where the perception of form and motion interact (Vaina, Solomon, Chowdhury, Sinha, \& Belliveau, 2001; Oram \& Perrett, 1996). In the STS, both facial and hand movements are analyzed according to their social significance (Allison et al., 2000). The STS receives axons from and sends projections into the amygdala and the VLPFC (Rolls, 2000; Puce, Allison, \& McCarthy, 1999). This tight interconnection and the highly important role of the STS for the recognition of emotional facial expressions (Iidaka et al., 2001; Hariri,
Bookheimer, \& Mazziotta, 2000; Breiter et al., 1996) make this region a key area for the recognition of emotional gestures. In our prestudy (Lotze et al., 2006), we observed a highly positive correlation between activation in the right STS and the negative valence of emotional gestures. In this context, an activation deficit in PD patients might result in a substantial deficit in the recognition of emotional gestures.

Consistent with other studies presenting emotional facial expressions in healthy individuals, we recorded activation during emotional gesture observation in both groups in areas related to recognition of faces and facial expression, such as the inferior temporal gyrus including the lateral fusiform gyrus (Kanwisher, McDermott, \& Chun, 1997), the inferior occipital gyrus (Hoffman \& Haxby, 2000), the STS, and the anterior temporal pole (Hoffman \& Haxby, 2000; Ishai, Ungerleider, Martin, \& Haxby, 2000). All these activation sites were decreased in PD patients as well as in areas related to emotional processing such as the amygdala, the orbito-frontal cortex, and the cingulate cortex (e.g., Ishai, Pessoa, Bikle, \& Ungerleider, 2004; Gorno-Tempini et al., 1998; for a recent overview, see Vuilleumier \& Pourtois, 2007). It has been demonstrated that increased emotional valence on presented faces enhance activation in visual cortical areas (Vuilleumier, Armony, Driver, \& Dolan, 2001; Critchley, Elliott, Mathias, \& Dolan, 2000; Breiter et al., 1996). Differences in attention of subject groups could not be excluded in this study. However, differences in mistakes of gesture recognition between subject groups were only significant for the emotional and not for the nonemotional task, suggesting that attention differences overall do not specifically influence activation maps for one type of stimuli.

Although patients ceased medication the night before the experiment $(\sim 12 \mathrm{hr})$, they were not completely in an off-state. Therefore, performance deficits and fMRI maps might have been affected by medication with longlasting effects. However, longer nonmedication periods are not tolerated by most of these patients.

Taken together, our data underscore recent findings that emotional processing is impaired in PD patients. This recognition deficit is correlated with dopamine transporter availability in the left putamen. Furthermore, our study opens a new view on possible interactions between dopaminergic loss in the putamen and access to ventrolateral prefrontal emotion processing areas, by showing correlative data between putamen DAT and BA 47 fMRI activation in PD patients with impaired gesture recognition. In addition, we demonstrated that the whole network involved in gesture recognition is less activated in PD patients including earlier and later visual processing; processing of facial and social recognition in the fusiform gyrus and the STS; areas involved in emotional processing such as the amygdala; areas associated with internal motor repetition such as BA 4, BA 44, and BA 6; and the ventrolateral prefrontal lobe. 


\section{Acknowledgments}

This study was supported by the DFG, SFB 550, and SFB 437. A. L. was supported by the Humboldt Foundation. We thank Nicola Neumann for corrections on the last version of the manuscript.

Reprint requests should be sent to Martin Lotze, Functional Imaging Group, Center for Diagnostic Radiology and Neuroradiology, University of Greifswald, Friedrich-Löffler-Straße 23a, D-17487 Greifswald, Germany, or via e-mail: martin.lotze@unigreifswald.de.

\section{REFERENCES}

Alexander, G. E., \& Crutcher, M. D. (1990). Functional architecture of basal ganglia circuits: Neural substrates of parallel processing. Trends in Neurosciences, 13, 266-271.

Allison, T., Puce, A., \& McCarthy, G. (2000). Social perception from visual cues: Role of the STS region. Trends in Cognitive Sciences, 4, 267-278.

Anders, S., Lotze, M., Erb, M., Grodd, W., \& Birbaumer, N. (2004). Brain activity underlying emotional valence and arousal: A response-related fMRI study. Human Brain Mapping, 23, 200-209.

Beck, A. T., Ward, C. H., Mendelson, M., Mock, J., \& Erbaugh, J. (1961). An inventory for measuring depression. Archives of General Psychiatry, 4, 561-571.

Bernheimer, H., Birkmayer, W., Hornykiewicz, O.,

Jellinger, K., \& Seitelberger, F. (1973). Brain dopamine and the syndromes of Parkinson and Huntington. Clinical, morphological and neurochemical correlations. Journal of the Neurological Sciences, 20, 415-455.

Blonder, L. X., Gur, R. E., \& Gur, R. C. (1989). The effects of right and left hemiparkinsonism on prosody. Brain and Language, 36, 193-207.

Braak, H., Braak, E., Yilmazer, D., de Vos, R. A., Jansen, E. N., \& Bohl, J. (1996). New aspects of pathology in Parkinson's disease with concomitant incipient Alzheimer's disease. Journal of Neural Transmission, Supplement, 48, 1-6.

Breit, S., Reimold, M., Reischl, G., Klockgether, T., \& Wullner, U. (2006). [(11)C]D-threo-methylphenidate PET in patients with Parkinson's disease and essential tremor. Journal of Neural Transmission, 113, 187-193.

Breitenstein, C., Daum, I., \& Ackermann, H. (1998). Emotional processing following cortical and subcortical brain damage: Contribution of the fronto-striatal circuitry. Behavioural Neurology, 11, 29-42.

Breitenstein, C., Van Lancker, D., Daum, I., \& Waters, C. H. (2001). Impaired perception of vocal emotions in Parkinson's disease: Influence of speech time processing and executive functioning. Brain and Cognition, 45, 277-314.

Breiter, H. C., Etcoff, N. L., Whalen, P. J., Kennedy, W. A., Rauch, S. L., Buckner, R. L., et al. (1996). Response and habituation of the human amygdala during visual processing of facial expression. Neuron, 17, 875-887.

Calder, A. J., Keane, J., Manes, F., Antoun, N., \& Young, A. W. (2000). Impaired recognition and experience of disgust following brain injury. Nature Neuroscience, 3, 1077-1078.

Calder, A. J., Lawrence, A. D., \& Young, A. W. (2001). Neuropsychology of fear and loathing. Nature Reviews Neuroscience, 2, 352-363.

Cavada, C., Company, T., Tejedor, J., Cruz-Rizzolo, R. J., \& Reinoso-Suarez, F. (2000). The anatomical connections of the macaque monkey orbitofrontal cortex. A review. Cerebral Cortex, 10, 220-242.
Cheung, C. C., Lee, T. M., Yip, J. T., King, K. E., \& Li, L. S. (2006). The differential effects of thalamus and basal ganglia on facial emotion recognition. Brain and Cognition, $61,262-268$

Critchley, H. D., Elliott, R., Mathias, C. J., \& Dolan, R. J. (2000). Neural activity relating to generation and representation of galvanic skin conductance responses: A functional magnetic resonance imaging study. Journal of Neuroscience, 20, 3033-3040.

Dewick, H. C., Hanley, J. R., Davies, A. D., Playfer, J., \& Turnbull, C. (1991). Perception and memory for faces in Parkinson's disease. Neuropsychologia, 29, 785-802.

Dubois, B., \& Pillon, B. (1997). Cognitive deficits in Parkinson's disease. Journal of Neurology, 244, 2-8.

Eickhoff, S. B., Stephan, K. E., Mohlberg, H., Grefkes, C., Fink, G. R., Amunts, K., et al. (2005). A new SPM toolbox for combining probabilistic cytoarchitectonic maps and functional imaging data. Neuroimage, 25, 1325-1335.

Fahn, S., \& Elton, R. L. (1987). Unified Parkinson Disease Rating Scale. New York: Macmillan.

Folstein, M. F., Folstein, S. E., \& McHugh, P. R. (1975). "Mini-mental state". A practical method for grading the cognitive state of patients for the clinician. Journal of Psychiatric Research, 12, 189-198.

Gatley, S. J., Ding, Y. S., Volkow, N. D., Chen, R., Sugano, Y., \& Fowler, J. S. (1995). Binding of D-threo-[11C]methylphenidate to the dopamine transporter in vivo: Insensitivity to synaptic dopamine. European Journal of Pharmacology, 281, 141-149.

Genovese, C. R., Lazar, N. A., \& Nichols, T. (2002). Thresholding of statistical maps in functional neuroimaging using the false discovery rate. Neuroimage, 15, 870-878.

Gorno-Tempini, M. L., Price, C. J., Josephs, O., Vandenberghe, R., Cappa, S. F., Kapur, N., et al. (1998). The neural systems sustaining face and proper-name processing. Brain, 121, 2103-2118.

Hariri, A. R., Bookheimer, S. Y., \& Mazziotta, J. C. (2000). Modulating emotional responses: Effects of a neocortical network on the limbic system. NeuroReport, 11, 43-48.

Haxby, J. V., Hoffman, E. A., \& Gobbini, M. I. (2000). The distributed human neural system for face perception. Trends in Cognitive Sciences, 4, 223-233.

Hennenlotter, A., Schroeder, U., Erhard, P., Haslinger, B., Stahl, R., Weindl, A., et al. (2004). Neural correlates associated with impaired disgust processing in pre-symptomatic Huntington's disease. Brain, 127, $1446-1453$.

Hirtz, D., Thurman, D. J., Gwinn-Hardy, K., Mohamed, M., Chaudhuri, A. R., \& Zalutsky, R. (2007). How common are the "common" neurologic disorders? Neurology, 68, 326-337.

Hoehn, M. M., \& Yahr, M. D. (1967). Parkinsonism: Onset, progression and mortality. Neurology, 17, 427-442.

Hoffman, E. A., \& Haxby, J. V. (2000). Distinct representations of eye gaze and identity in the distributed human neural system for face perception. Nature Neuroscience, 3, $80-84$.

Hornak, J., Rolls, E. T., \& Wade, D. (1996). Face and voice expression identification in patients with emotional and behavioural changes following ventral frontal lobe damage. Neuropsychologia, 34, 247-261.

Iidaka, T., Omori, M., Murata, T., Kosaka, H., Yonekura, Y., Okada, T., et al. (2001). Neural interaction of the amygdala with the prefrontal and temporal cortices in the processing of facial expressions as revealed by fMRI. Journal of Cognitive Neuroscience, 13, 1035-1047.

Innis, R. B., Cunningham, V. J., Delforge, J., Fujita, M., Gjedde A., Gunn, R. N., et al. (2007). Consensus nomenclature for 
in vivo imaging or reversibly binding radioligands. Journal of Cerebral Blood Flow and Metabolism, 27, 1533-1539.

Ishai, A., Pessoa, L., Bikle, P. C., \& Ungerleider, L. G. (2004). Repetition suppression of faces is modulated by emotion. Proceedings of the National Academy of Sciences, U.S.A., 101, 9827-9832.

Ishai, A., Ungerleider, L. G., Martin, A., \& Haxby, J. V. (2000). The representation of objects in the human occipital and temporal cortex. Journal of Cognitive Neuroscience, 12(Suppl. 2), 35-51.

Kan, Y., Kawamura, M., Hasegawa, Y., Mochizuki, S., \& Nakamura, K. (2002). Recognition of emotion from facial, prosodic and written verbal stimuli in Parkinson's disease. Cortex, 38, 623-630.

Kanwisher, N., McDermott, J., \& Chun, M. M. (1997). The fusiform face area: A module in human extrastriate cortex specialized for face perception. Journal of Neuroscience, 17, 4302-4311.

Lawrence, A. D., Calder, A. J., McGowan, S. W., \& Grasby, P. M. (2002). Selective disruption of the recognition of facial expressions of anger. NeuroReport, 13, 881-884

Lawrence, A. D., Goerendt, I. K., \& Brooks, D. J. (2007). Impaired recognition of facial expressions of anger in Parkinson's disease patients acutely withdrawn from dopamine replacement therapy. Neuropsychologia, 45, 65-74.

Levesque, J., Eugene, F., Joanette, Y., Paquette, V., Mensour, B., Beaudoin, G., et al. (2003). Neural circuitry underlying voluntary suppression of sadness. Biological Psychiatry, 53, 502-510.

Logan, J., Fowler, J. S., Volkow, N. D., Wang, G. J., Ding, Y. S., \& Alexoff, D. L. (1996). Distribution volume ratios without blood sampling from graphical analysis of PET data. Journal of Cerebral Blood Flow and Metabolism, 16, 834-840.

Logothetis, N. K., Guggenberger, H., Peled, S., \& Pauls, J. (1999). Functional imaging of the monkey brain. Nature Neuroscience, 2, 555-562.

Lotze, M., Heymans, U., Birbaumer, N., Veit, R., Erb, M., Flor, H., et al. (2006). Differential cerebral activation during observation of expressive gestures and motor acts. Neuropsychologia, 44, 1787-1795.

Middleton, F. A., \& Strick, P. L. (1996). The temporal lobe is a target of output from the basal ganglia. Proceedings of the National Academy of Sciences, U.S.A., 93, 8683-8687.

Oldfield, R. C. (1971). The assessment and analysis of handedness: The Edinburgh inventory. Neuropsychologia, 9, 97-113.

Oram, M. W., \& Perrett, D. I. (1996). Integration of form and motion in the anterior superior temporal polysensory area (STPa) of the macaque monkey. Journal of Neurophysiology, 76, 109-129.

Pell, M. D. (1996). On the receptive prosodic loss in Parkinson's disease. Cortex, 32, 693-704.

Phan, K. L., Wager, T., Taylor, S. F., \& Liberzon, I. (2002). Functional neuroanatomy of emotion: A meta-analysis of emotion activation studies in PET and fMRI. Neuroimage, 16, 331-348.

Phillips, M. L., Young, A. W., Scott, S. K., Calder, A. J., Andrew, C., Giampietro, V., et al. (1998). Neural responses to facial and vocal expressions of fear and disgust. Proceedings of the Royal Society of London, Series B, Biological Sciences, 265, 1809-1817.

Puce, A., Allison, T., \& McCarthy, G. (1999). Electrophysiological studies of human face perception: III. Effects of top-down processing on face-specific potentials. Cerebral Cortex, 9, 445-458.
Rolls, E. T. (2000). Precis of the brain and emotion. Behavioral and Brain Sciences, 23, 177-191; discussion 192-233.

Sato, T., Takahashi, N., \& Kawamura, M. (2008). Impaired recognition of emotion improved by L-DOPA in a patient with amygdala damage. Rinsho Shinkeigaku, 48, 139-142.

Shmuelof, L., \& Zohary, E. (2005). Dissociation between ventral and dorsal fMRI activation during object and action recognition. Neuron, 47, 457-470.

Smith, M. C., Smith, M. K., \& Ellgring, H. (1996). Spontaneous and posed facial expression in Parkinson's disease. Journal of the International Neuropsychological Society, 2, 383-391.

Sossi, V., de la Fuente-Fernández, R., Schulzer, M., Troiano, A. R., Ruth, T. J., \& Stoessi, A. J. (2007). Dopamine transporter relation to dopamine turnover in Parkinson's disease: A positron emission tomography study. Annals of Neurology, 62, 468-474.

Sprengelmeyer, R., Rausch, M., Eysel, U. T., \& Przuntek, H. (1998). Neural structures associated with recognition of facial expressions of basic emotions. Proceedings of the Royal Society of London, Series B, Biological Sciences, 265, 1927-1931.

Sprengelmeyer, R., Young, A. W., Mahn, K., Schroeder, U., Woitalla, D., Buttner, T., et al. (2003). Facial expression recognition in people with medicated and unmedicated Parkinson's disease. Neuropsychologia, 41, 1047-1057.

Suzuki, A., Hoshino, T., Shigemasu, K., \& Kawamura, M. (2006). Disgust-specific impairment of facial expression recognition in Parkinson's disease. Brain, 129, 707-717.

Tessitore, A., Hariri, A. R., Fera, F., Smith, W. G., Chase, T. N., Hyde, T. M., et al. (2002). Dopamine modulates the response of the human amygdala: A study in Parkinson's disease. Journal of Neuroscience, 22, 9099-9103.

Tzourio-Mazoyer, N., Landeau, B., Papathanassiou, D., Crivello, F., Etard, O., Delcroix, N., et al. (2002). Automated anatomical labeling of activations in SPM using a macroscopic anatomical parcellation of the MNI MRI single-subject brain. Neuroimage, 15, 273-289.

Vaina, L. M., Solomon, J., Chowdhury, S., Sinha, P., \& Belliveau, J. W. (2001). Functional neuroanatomy of biological motion perception in humans. Proceedings of the National Academy of Sciences, U.S.A., 98, 11656-11661.

Volkow, N. D., Ding, Y. S., Fowler, J. S., Wang, G. J., Logan, J., Gatley, S. J., et al. (1995). A new PET ligand for the dopamine transporter: Studies in the human brain. Journal of Nuclear Medicine, 36, 2162-2168.

Vuilleumier, P., Armony, J. L., Driver, J., \& Dolan, R. J. (2001) Effects of attention and emotion on face processing in the human brain: An event-related fMRI study. Neuron, 30, 829-841.

Vuilleumier, P., \& Pourtois, G. (2007). Distributed and interactive brain mechanisms during emotion face perception: Evidence from functional neuroimaging. Neuropsychologia, 45, 174-194.

Wildgruber, D., Hertrich, I., Riecker, A., Erb, M., Anders, S., Grodd, W., et al. (2004). Distinct frontal regions subserve evaluation of linguistic and emotional aspects of speech intonation. Cerebral Cortex, 14, 1384-1389.

Wildgruber, D., Riecker, A., Hertrich, I., Erb, M., Grodd, W., Ethofer, T., et al. (2005). Identification of emotional intonation evaluated by fMRI. Neuroimage, 24, 1233-1241.

Yin, H. H., \& Knowlton, B. J. (2006). The role of the basal ganglia in habit formation. Nature Reviews Neuroscience, 7, 464-476. 